\title{
Diagnostic test for ion implantation dosimetry
}

\author{
S. Matteson, D. G. Tonn, and M-A. Nicolet \\ California Institute of Technology, Pasadena, California 91125 \\ (Received 8 February 1979; accepted 27 March 1979)
}

A diagnostic technique is discussed and illustrated by experiment, which reveals sources of error in current integration dosimetry. The technique uses simple, specially prepared samples and an oscilloscope display of the measured current versus time.

PACS numbers: $61.70 . \mathrm{Tm}, 85.30 .-\mathrm{z}, 07.50 .+\mathrm{f}$

\section{l. INTRODUCTION}

Ion implantation has become an accepted tool in the fabrication of solid-state devices. Two reasons for the widespread use of ion implantation are its potential for precise doping control and for reproducibility. However, effects peculiar to ion impact on solids can interfere with accurate dosimetry and thereby jeopardize these primary advantages of ion implantation. Diagnostic techniques are therefore needed to determine the presence and origin of dosimetry errors. The most straightforward method of dosimetry is the integration of the ion current falling on the sample. ${ }^{1}$ We present here a simple test that diagnoses errors in such an integration system.

From the total charge deposited on the target, one can easily calculate the total number of incident ions that have been implanted if the average charge state of the ions is known. Complications arise, however, because secondary electrons and ions, which are generated by the primary particles impacting the sample and other surfaces, can cause spurious currents which are also integrated. The number and type of secondary particles depends on the incident ions' species, the sample material, and on the surface condition of the sample. ${ }^{2}$ The problem may be complicated further by the action of suppression biases applied to various electrodes in the vicinity of the target. Frequently, these suppression biases are only partially effective in suppressing the various secondary particles. ${ }^{3}$

A diagnostic procedure should reveal the presence and magnitude of spurious current. The test should permit immediate evaluation of modifications and adjustments of suppression biases, and the procedure should require very little in the way of ancillary equipment or ion implantation modification. These criteria are met in our test by preparing target samples which generate a higher yield of secondary particles over one half of the surface while the other half generates the normal current of secondary particles. The current in the sample is monitored by an oscilloscope synchronized to the sweep of the ion beam across the target. The change in the sample current as the primary beam is scanned over the two halves measures the relative magnitude of the secondary error current.

We prepared two types of samples and both performed well. The first is a silicon single-crystal wafer upon half of whose face was evaporated $500 \AA$ of gold. The gold film has a higher secondary electron yield than silicon. The second type of sample is a 2-in. tantalum disk half of which is covered by a tantalum "roof top," i.e., an inverted " $V$ " of Ta sheet. This sample makes use of the fact that the emission of secondary electrons and ions strongly varies with the angle of incidence of the primary particle. Over one half of the tantalum disk the ions are incident nearly perpendicularly to the surface, while over the other half they penetrate at a grazing angles. The targets are sketched on the upper left sides of Figs. 1 and 2.

\section{EXPERIMENTAL TECHNIQUE}

Figures 1 and 2 illustrate the use of these test samples. The same charge collection arrangement was used in both cases. The electrodes labeled A and B are electrically isolated from the target chamber. Any current flowing through them must pass the integrator too or, in the present case, through the oscilloscope input (represented in the figures by the circle with the schematic waveform). The electrodes A and B together form a Faraday cup. A grounded electrode (labeled "window") defines the beam scan area and a second electrode biased at $-500 \mathrm{~V}$ dc (labeled "suppressor") is located in the shadow of the window and suppresses the emission of secondary electrons from the edge of the window into the Faraday cup. Figures 1 and 2 show photographs of the oscilloscope trace for the three bias arrangements [labeled (a), (b), and (c)] sketched in the corresponding schematics. The traces represent the measured current versus time or, equivalently, versus position. The incident ion was $100 \mathrm{kV} \mathrm{Kr}^{+}$in all cases.

The bias arrangements were chosen to illustrate common sources of error in current integration and their identification by the present diagnostic test. As can be seen in the trace (a), there is a $15 \%$ or $50 \%$ increase in the current when the beam impinges on the $\mathrm{Au}$ or on the inclined section of the target. This spurious increase can be explained by a loss of secondary electrons generated within the Faraday cup (A) to the vacuum vessel walls.

In part (b) the polarity of the Faraday cup (A) bias is reversed and the target is positive with respect to electrode $B$ which is held near earth potential via the oscilloscope input. Now the escape of secondary electrons from the target is suppressed electrostatically and the error current is reduced, but a new complication arises. Secondary positive ions or energetic neutral particles which leave the Faraday cup will produce electrons (which we shall call "tertiary electrons") at any solid surface which they encounter. The measured current will reflect both the loss of positive ions and the col- 


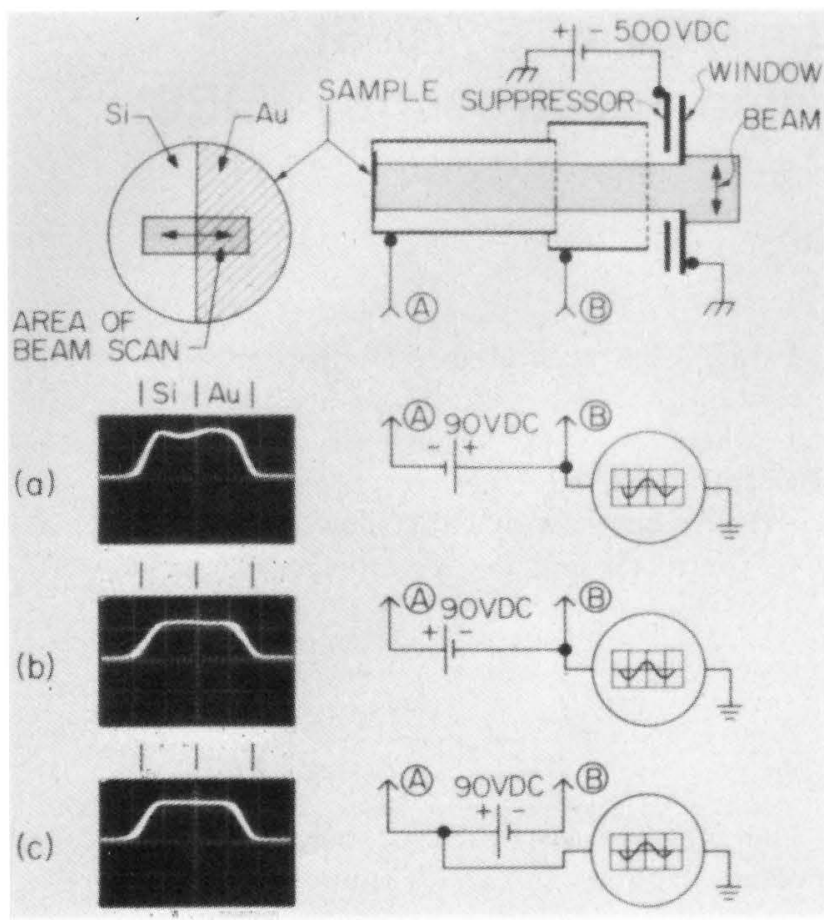

FIG. 1. Schematic drawing of Si-Au test sample and current integration arrangement. Electrodes $A$ and $B$ are electrically isolated from ground and connected to the corresponding labeled points $A$ and $B$ in three biasing schemes. The circle with the raster represents an oscilloscope with a $1 \mathrm{M} \Omega$ input resistance. The scan area is defined by the aperture labeled window, and secondary electrons arising from the edge of the window are suppressed by the electrode labeled suppressor. The oscilloscope traces (current versus position) which were observed are shown next to the corresponding set up schematic. The vertical and horizontal scales are the same for all traces. $(5 \mu \mathrm{A} / \mathrm{div}$ and $1 \mathrm{~ms} / \mathrm{div}$ or $0.4 \mathrm{in} . / \mathrm{div})$.

lection of tertiary electrons by a decrease in relative magnitude during the time the beam is incident on the Au or inclined part of the test samples. The presence of tertiary electrons and their role in ion beam measurement is discussed at length in a paper by Matteson et al. ${ }^{4}$ The traces labeled (b) show this change in the current as a decrease of about $5 \%$ and $10 \%$.

In (c) the two electrodes A and B have the same bias with respect to each other as in (b), but a change in their potential with respect to the ground (i.e., the vacuum chamber walls) was made. The result is good suppression of secondary electrons and negative ions with an improvement in the collection of secondary positive ions by the electrode $\mathrm{B}$. In the case of the Si-Au sample (Fig. 1), no detectable change is noted to within an experimental error of about $2 \%$. In the other case (Fig. 2), the current increases by $5 \%$ which is opposite to the change observed in part (b). This increase could be caused by the loss to the vacuum chamber wall of secondary electrons produced by sputtered Ta atoms impacting on electrode $\mathrm{B}$. Taking into account the strong enhancement of sputtering at slanted angles of beam incidence (proportional to $\cos ^{-1}$ ), this increase corresponds to less than a $2 \%$ error for a Ta target at

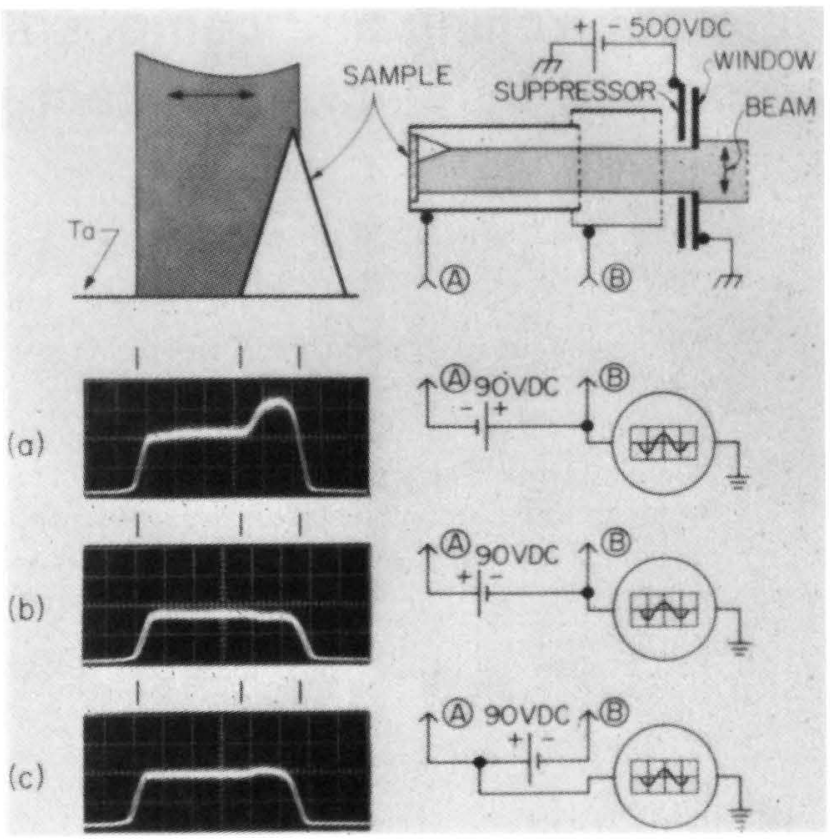

FIG. 2. Schematic drawing of Ta test sample and current integration arrangements. The symbols are as defined for Fig. 1. The sample consists of a Ta disk onto which is welded an inverted Ta " $\mathrm{V}$ ". As the beam is swept across the sample, the secondary emission processes are strongly enhanced as the angle of incidence changes from perpendicular to glancing. The resulting change in the measured current is indicative of the magnitude and sense of spurious currents.

normal incidence. We have applied this test on a commercial target assembly, and found that errors of more than $20 \%$ easily occur.

\section{CONCLUSION}

The diagnostic technique described can be easily used to test the effectiveness of a conventional charge integration system employed in ion implantation systems. The test samples are simple to prepare. Spurious currents of a few percent can be detected, depending on the actual geometry of $\mathrm{A}$ and $\mathrm{B}$ and the ground wall. Thus, the test procedure provides a simple, prompt, and accurate way to check ion implantation dosimetry for errors due to secondary emission effects.

\section{ACKNOWLEDGMENTS}

The authors gratefully acknowledge the support of $\mathrm{R}$. Gorris and J. Mallory for the preparation of the diagnostic samples. This research was supported in part by the U.S. Army Research Office under the Joint Services Electronic Program (DAAG-29-77-C-0015)

${ }^{1}$ G. Dearnaley, J. H. Freeman, R. S. Nelson, and J. Stephen, Ion Implantation (Elsevier, New York, 1973), p. 476.

${ }^{2}$ H. S. Hagstrum, Rev. Sci. Instrum. 24, 1136 (1953).

${ }^{3}$ D. M. Jamba, Rev. Sci. Instrum. 49, 643 (1978).

${ }^{4} \mathrm{~S}$. Matteson and M-A. Nicolet, Nucl. Instrum. Methods (in press). 